\title{
PERFIL DE MANIFESTAÇÃO DE ESTRESSE EM UNIVERSITÁRIOS: UM ESTUDO TRANSVERSAL
}

\section{PROFESSION OF STRESS EXPRESSION IN UNIVERSITY STUDENTS: A CROSS-SECTION STUDY}

\author{
Miqueline Mendonça Martins Lima ${ }^{1} *$ Janiel Ferreira Felício $^{2} *$ Alberto João M'batna $^{3} *$ Diego da \\ Silva Ferreira $^{4} *$ Mariana Carvalho e Souza Leão Cavalcante ${ }^{5}$. Francisco Clécio da Silva Dutra $^{6} *$ \\ Lydia Vieira Freitas dos Santos ${ }^{7} *$ Aline Santos Monte ${ }^{8}$ Cinthia Mendonça Cavalcante ${ }^{9} *$ Jeferson $^{*}$ \\ Falcão do Amaral ${ }^{10}$
}

\begin{abstract}
RESUMO
Objetivo: avaliar o perfil dos diferentes estágios da manifestação de estresse em discentes de uma Instituição Federal de Ensino Superior (IFES), no município de Redenção-CE, refletindo e discutindo o possível impacto disto no processo de aprendizagem e na qualidade de vida dos mesmos. Métodos: estudo realizado com discentes de uma IFES, ambos os sexos, brasileiros e estrangeiros, matriculados em diferentes cursos de graduação da instituição. As descrições sobre as manifestações de estresse foram realizadas através do Inventário de Estresse de Lipp (ISS). Resultados: foram coletados dados de 120 alunos regularmente matriculados em diferentes cursos de graduação, modalidade presencial, nos Campi da IFES localizados no Estado do Ceará. Observou-se que cerca de $94(78,4 \%)$ apresentaram sintomas da manifestação de estresse em algumas das fases apontadas no instrumento utilizado. Enquanto $26(21,6 \%)$ não apresentaram quaisquer dos sintomas. Conclusão: evidenciou-se que as manifestações de estresse dos discentes participantes foram consideravelmente altas. Diante disto, denota-se a necessidade de se trabalhar melhor os pontos relacionados ao estresse no ambiente universitário, com vistas a diminuir os impactos biopsicossociais acarretados pela rotina da vida acadêmica.
\end{abstract}

Palavras-chave: Estresse Ocupacional; Aprendizagem; Estudantes; Universidades.

\section{ABSTRACT}

Objective: to evaluate the profile of the different stages of the manifestation of stress in students of a Federal Institution of Higher Education (FIHE), in the municipality of Redenção-CE, reflecting and discussing the possible impact of this on their learning process and their quality of life. Methods: a study carried out with IFES students, both sexes, Brazilian and foreign, enrolled in different undergraduate courses at the institution. Descriptions of the manifestations of stress were carried out through the Lipp Stress Inventory (ISS). Results: data were collected from 120 students regularly enrolled in different undergraduate courses, in person, at FIHE Campi located in the State of Ceará. It was observed that about 94 (78.4\%) showed symptoms of stress in some of the phases indicated in the instrument used. While 26 (21.6\%) did not show any of the symptoms. Conclusion: it was shown that the stress manifestations of the participating students were considerably high. In view of this, there is a need to better work on points related to stress in the university environment, with a view to reducing the biopsychosocial impacts caused by the routine of academic life.

Keywords: Occupational Stress; Learning; Students; Universities.

\footnotetext{
${ }^{1}$ Enfermeira pela Universidade da Integração Internacional da Lusofonia Afro-Brasileira (UNILAB).

${ }^{2}$ Acadêmico de Enfermagem na Universidade da Integração Internacional da Lusofonia Afro-Brasileira (UNILAB).

${ }^{3}$ Enfermeiro. Mestrando em Sociobiodiversidade e Tecnologias Sustentáveis pela Universidade da Integração Internacional da Lusofonia AfroBrasileira (UNILAB).

${ }^{4}$ Enfermeiro. Especialista em Saúde da Família. Mestre em Enfermagem pela Universidade da Integração Internacional da Lusofonia Afro-Brasileira (UNILAB). Doutorando em Saúde Coletiva na Universidade Estadual do Ceará (UECE).

${ }^{5}$ Enfermeira. Mestre e Doutora em Ciências da Saúde pela Universidade Estadual de Campinas - FEN/ UNICAMP. Docente na Universidade da Integração Internacional da Lusofonia Afro-Brasileira (UNILAB).

${ }^{6}$ Enfermeiro. Biólogo. Especialista em Saúde da Família pela Universidade da Integração Internacional da Lusofonia Afro-Brasileira (UNILAB). Mestre e Doutorando em Cuidados Clínicos em Enfermagem e Saúde pela Universidade Estadual do Ceará (UECE).

${ }^{7}$ Enfermeira. Mestre e Doutora em Enfermagem pela Universidade Federal do Ceará (UFC). Docente na Universidade da Integração Internacional da Lusofonia Afro-Brasileira (UNILAB).

${ }^{8}$ Farmacêutica. Mestre e Doutora em Farmacologia pela Universidade Federal do Ceará (UFC). Docente na Universidade da Integração Internacional da Lusofonia Afro-Brasileira (UNILAB).

${ }^{9}$ Psicóloga. Mestre em Saúde Pública e Doutora em Saúde Coletiva pela Universidade Estadual do Ceará (UECE). Pós Doutoramento em parceria com a University of Birmingham (Brazillian Fellowship). Docente na Universidade Federal do Ceará (UFC).

${ }^{10}$ Farmacêutico Clínico. Mestre e Doutor em Farmacologia pela Universidade Federal do Ceará (UFC). Docente na Universidade da Integração Internacional da Lusofonia Afro-Brasileira (UNILAB).
} 


\section{INTRODUÇÃO}

O estresse é definido como uma resposta física desencadeada por estímulos internos e/ou externos; e é considerado um mecanismo de defesa. Denomina-se estímulo externo aquele que está relacionado aos hábitos de vida e ao ambiente, já o estímulo interno refere-se à personalidade e a maneira como o indivíduo vive $\mathrm{e}^{(1)}$.

Um conjunto de reações fisiológicas manifestam-se no momento em que a pessoa está sob tensão, pressão e insistência. Quando o cérebro entende que algum evento é ameaçador, ele começa a dar uma resposta ao organismo em forma de sintomas físicos ou psicológicos, esse mecanismo é denominado estresse; considerado um problema de saúde pública que está presente em $90 \%$ na população mundial $^{(2)}$. O corpo humano possui diversos hormônios, e alguns desses são ativados quando o indivíduo se vê diante de um fator estressor; é através desta ativação hormonal que é desencadeado o estresse ${ }^{(3)}$.

A somatização é uma psicopatologia em que o indivíduo apresenta sintomas físicos, cujas queixas vêm acompanhadas de alterações clinicamente constatáveis que são causadas ou intensificadas por razões emocionais ${ }^{(4)}$. Alguns autores descrevem que a redução na qualidade de vida, queda na produtividade, perda de dias de trabalho e/ou escolares, além de maiores custos aos sistemas de saúde, principalmente quando há associação de estresse e distúrbios vida universitária, já precisa lidar com frustrações, temores, angústias e ainda mudar psicossomáticos, são fatores que podem ser minimizados quando os sinais de estresse são identificados e o encaminhamento e tratamento são realizados precocemente ${ }^{(5)}$.

Pagliarone e Sforcin, ${ }^{(6)}$ descrevem a resposta biológica a eventos estressores como Síndrome da Adaptação Geral ao Estresse; ou seja, quando o cérebro detecta que o indivíduo entrou em contato com algum fator estressante o corpo começa a reagir e o organismo passa a ter uma série de reações levando a um processo de adaptação. Esse processo possui três fases consecutivas: fase 1 (alerta), fase 2 (resistência) e fase 3 (exaustão) $^{(7)}$.

$\mathrm{O}$ estresse pode ser positivo ou negativo. Quando positivo, o ser humano consegue centrar-se nos seus objetivos e, mesmo sob pressão, consegue atingir suas metas e ter bons resultados nas suas atividades de vida diária; este evento é denominado eustresse. $\mathrm{O}$ estresse negativo ou distresse, por sua vez, ocorre quando a pessoa já está esgotada e ultrapassa seus limites; o organismo adoece e sua saúde fica prejudicada. Neste caso, o indivíduo começa a apresentar sintomas físicos como: fadiga crônica, dor nas costas, dor de cabeça e alteração no apetite ${ }^{(8)}$.

A fase de iniciação à vida universitária é considerada como um fator extremamente estressante, pois o aluno ingresso passou muito tempo se preparando para entrar em uma universidade e, após os primeiros meses de seus hábitos de vida de forma súbita podendo desenvolver uma série de reações 
psicofisiológicas $^{(9)}$.

Os sintomas do estresse estão presentes em estudantes universitários, especialmente naqueles que estão ingressando na universidade e têm que mudar a sua rotina repentinamente de forma a adequar-se ao calendário acadêmico. Juntamente com o ingresso do aluno na universidade, associa-se a uma série de sentimentos e emoções que podem ser denominados como fatores estressores $^{(1)}$.

Dessa forma, considerando ser frequente a realidade do estresse na dimensão acadêmica e sociocultural dos discentes, o presente estudo objetivou avaliar o perfil dos diferentes estágios da manifestação de estresse em discentes de uma Instituição Federal de Ensino Superior (IFES), no município de Redenção-CE, refletindo e discutindo o possível impacto disto no processo de aprendizagem e na qualidade de vida dos mesmos.

\section{MATERIAIS E MÉTODOS}

Trata-se de um estudo transversal e descritivo com abordagem quantitativa, realizado no período de julho de 2017 a julho de 2018 com acadêmicos de uma IFES localizada no município de Redenção - CE. É uma instituição de ensino que, em sua política, promove integração entre países de Língua Portuguesa oficial, dividida em
Institutos em diferentes Campi, onde todos os cursos estão alocados dentro dos mesmos.

Adotou-se como critério de inclusão os alunos estarem devidamente matriculados nos cursos de graduação. O cálculo amostral com nível de confiança de $95 \%$, delimitou uma amostra de 327 discentes; levando em consideração um total de 2176 alunos matriculados nos cursos. Após aplicação dos critérios de inclusão e leitura do TCLE, apenas 120 discentes concordaram e participaram da pesquisa. Considerando que os alunos matriculados nos cursos semipresenciais, pós-graduação Lato sensu e Stricto sensu possuem seus horários de atividade acadêmicas extremamente aleatórias e que muitos estudam na modalidade à distância, percebeu-se que estes não vivenciam de forma plena o dia a dia da rotina universitária, por isso os mesmos foram excluídos do estudo.

A coleta de dados foi iniciada após assinatura do Termo de Consentimento Livre e Esclarecido (TCLE) e se deu por meio de dois questionários: o primeiro versava sobre o perfil socioeconômico e demográfico e o segundo sobre as manifestações do estresse relatadas pelos discentes durante as entrevistas pelo instrumento de LIPP.

\section{Quadro 01 - Critérios de avaliação do inventário de Lipp}




\begin{tabular}{|l|l|l|l|} 
& $\begin{array}{l}\text { Sintomas } \\
\text { listados nas } \\
\text { últimas 24 horas }\end{array}$ & $\begin{array}{l}\text { Sintomas } \\
\text { listados } \\
\text { no último mês }\end{array}$ & $\begin{array}{l}\text { Sintomas } \\
\text { listados nos } \\
\text { últimos 3 meses }\end{array}$ \\
\hline Apresenta estresse & $\geq 7$ & $\geq 4$ & $\geq 9$ \\
\hline
\end{tabular}

Fonte: Lipp \& Tanganelli; 2002.

O Inventário de Sintomas de Estresse (ISS) consiste em um instrumento de verificação de sintomas físicos e psicológicos relacionados ao estresse, divididos em três partes (ver figura 01). Na primeira parte, o respondente deve indicar sintomas que experimentou nas últimas 24 horas correspondente a fase 1 (alerta) que ocorre quando se tem contato com o agente estressor e o corpo se prepara naturalmente para luta ou fuga gerando uma série de mudanças fisiológicas; na segunda parte os sintomas que experimentou no último mês que refere-se a fase 2 (resistência), ocorre quando há uma tentativa de reequilíbrio do organismo, nesta etapa há um grande gasto de energia o que deixa os sintomas visíveis; e na terceira parte, os sintomas experimentados nos últimos três meses, que corresponde à fase 3 (exaustão), neste período o corpo não consegue voltar ao seu estado normal após um momento de estresse. Durante este episódio as doenças começam a se manifestar. Todos esses sintomas listados no instrumento são referentes aos sintomas físicos e psicológicos. O ISS permite demonstrar se a pessoa tem estresse, em que fase ela se encontra e se sua sintomatologia é mais típica da área somática ou cognitiva ${ }^{(10)}$.

https://doi.org/10.31011/reaid-2021-v.95-n.33-art.903
Os universitários foram convidados a participar da pesquisa de forma aleatória nos horários de intervalos das aulas. Salienta-se que os discentes foram orientados sobre como responder o teste da seguinte forma: o Inventário de Sintomas de Estresse de Lipp consiste em um questionário com três quadros, todos com uma lista de sintomas físicos e psicológicos que devem ser marcados de acordo com o que você sentiu nas últimas 24 horas; no último mês e nos últimos 3 meses. Após a explicação, o instrumento autoaplicável foi entregue aos discentes. Neste estudo, as variáveis foram: dependentes (diferentes formas de manifestação de estresse) e as independentes (idade, sexo, estado civil, nacionalidade, residência atual, reprovação, bolsista de pesquisa/ extensão e atividade profissional).

Após a coleta, os dados foram organizados e o perfil da manifestação de estresse foi elaborado com o auxílio de tabelas. A análise dos dados foi realizada com a descrição de frequências absolutas e relativas, reflexão e discussão dos dados à luz da literatura vigente.

O estudo foi aprovado pelo Comitê de Ética em Pesquisa da UNILAB sob o parecer $\mathrm{n}^{\mathrm{o}}$. 1.435.776 e CAAE $\mathrm{n}^{\circ}$ Rev Enferm Atual In Derme v. 95, n. 33, 2021 e-021001 
49248215.9.0000.5576. O trabalho atendeu ao que preconiza a Resolução $\mathrm{n}^{\circ} 466 / 12$, do Conselho Nacional de Saúde (CNS), que configura os aspectos da autonomia, não- maleficência, beneficência e justiça, tudo isto em benefício e proteção ao usuário, à sociedade e ao Estado.

\section{$\underline{\text { Inventário de estresse de Lipp - ISS }}$}

Idade:

Sexo: ( ) Masc. ( ) Fem.

Semestre:

TESTE DE LIPP - Avaliação de Estresse

\begin{tabular}{|c|c|c|c|c|c|c|}
\hline \multirow{2}{*}{\multicolumn{3}{|c|}{$\begin{array}{r}\text { Coluna I.1 } \\
\text { Mäos } \mathrm{e} / \text { ou pés frios }\end{array}$}} & & & & \\
\hline 01 & & & & \multicolumn{3}{|c|}{$\begin{array}{c}\text { Coluna II.2 } \\
\text { Taquicardia (batimentos acelerados do coração) }\end{array}$} \\
\hline 02 & \multicolumn{3}{|l|}{$\begin{array}{l}\text { Māos e/ ou pés frios } \\
\text { Boca seca }\end{array}$} & 02 & \multicolumn{2}{|c|}{$\begin{array}{l}\text { Taquicardia (batimentos acelerados do coraçăo) } \\
\text { Respiraç̃o ofegante, entrecortada }\end{array}$} \\
\hline 03 & \multicolumn{3}{|l|}{ Nó ou dor no estômago } & 03 & \multicolumn{2}{|c|}{$\begin{array}{l}\text { Hipertensão súbita e passageira (pressão alta } \\
\text { súbita e passageira) }\end{array}$} \\
\hline 04 & \multicolumn{3}{|c|}{ Aumento de sudorese (muito suor) } & 04 & \multicolumn{2}{|c|}{$\begin{array}{l}\text { Mudança de apetite (comer bastante ou ter falta } \\
\text { de apetite) }\end{array}$} \\
\hline 05 & \multicolumn{3}{|c|}{$\begin{array}{l}\text { Tensão muscular (dor nas costas, pescoço, } \\
\text { ombros) }\end{array}$} & 05 & \multicolumn{2}{|c|}{ Aumento súbito de motivação } \\
\hline 06 & \multicolumn{3}{|c|}{$\begin{array}{l}\text { Aperto na Mandíbula, ranger de dente, roer as } \\
\text { unhas ou ponta da caneta }\end{array}$} & 06 & \multicolumn{2}{|c|}{ Entusiasmo súbito } \\
\hline 07 & \multicolumn{3}{|l|}{ Diarréia passageira } & 07 & \multirow{2}{*}{\multicolumn{2}{|c|}{ Vontade súbita de iniciar novos projetos }} \\
\hline 08 & \multicolumn{3}{|l|}{ Insönia, dificuldade de dormir } & & & \\
\hline \multicolumn{7}{|c|}{ Total de itens assinalados ( Soma das colunas l.1 e 1.2 . } \\
\hline \multicolumn{7}{|c|}{ II - Assinale os sintomas que tem experimento no ÚLTIMO MES - FASE II } \\
\hline \multicolumn{4}{|c|}{ Coluna II.1 } & \multicolumn{3}{|r|}{ Coluna II. 2} \\
\hline 01 & \multicolumn{3}{|c|}{ Problemas com memória, esquecimento } & 01 & \multicolumn{2}{|c|}{$\begin{array}{l}\text { Aparecimento de gastrite prolongada (queimação } \\
\text { no estômago, azia) }\end{array}$} \\
\hline 02 & \multicolumn{3}{|c|}{ Mal-estar generalizado, sem causa especifica } & 02 & \multicolumn{2}{|c|}{ Tontura, Sensaçäo de estar flutuando } \\
\hline 03 & \multicolumn{3}{|c|}{ Formigamento nasextremidades (pés ou mãos) } & 03 & \multicolumn{2}{|c|}{$\begin{array}{l}\text { Sensibilidade emotiva excessiva, emociona-se por } \\
\text { qualquer coisa }\end{array}$} \\
\hline 04 & \multicolumn{3}{|c|}{ Sensaçāo de desgaste fisico constante } & 04 & \multicolumn{2}{|c|}{ Dúvidas quanto a si próprio } \\
\hline 05 & \multicolumn{3}{|l|}{ Mudança de apetite } & 05 & \multicolumn{2}{|c|}{ Pensamento constante sobre só um assunto } \\
\hline 06 & \multicolumn{3}{|c|}{$\begin{array}{l}\text { Aparecimento de problemas dermatológicos } \\
\text { (pele) }\end{array}$} & 06 & Irritabilic & de excessiva \\
\hline 07 & Hipertensão arterial (episódio & dep & ssão alta) & 07 & Diminuiç & da libido (desejo sexual diminuido) \\
\hline 08 & Cansaço constante & & & & & \\
\hline & & & & & assinala & ( Soma das colunas 11.1 e 11.2 \\
\hline & $\frac{\text { Sinale os sintomas }}{\text { Coluna III.1. }}$ & & Coluna & & & Coluna III.3 3 \\
\hline 01 & Diarréias frequentes & 01 & Tonturat & quente & 1 & \begin{tabular}{|l} 
Impossibilidade de trabalhar \\
\end{tabular} \\
\hline 02 & Dificuldades sexuais & 02 & Úlcera & & 02 & $\begin{array}{l}\text { Sensação de incompetência em todas } \\
\text { as áreas }\end{array}$ \\
\hline 03 & $\begin{array}{l}\text { Formigamentos nas } \\
\text { extremidades (mãos e pés) }\end{array}$ & 03 & Infarto & & 03 & Vontade de fugir de tudo \\
\hline 04 & Insônia & 04 & Pesadelo & & 04 & $\begin{array}{l}\text { Apatia, vontade de nada fazer, } \\
\text { depressão ou raiva prolongadas }\end{array}$ \\
\hline 05 & Tiques nervosos & 05 & Cansaço & cessivo & 05 & $\begin{array}{l}\text { Taquicardia (batimento acelerado do } \\
\text { coracão) }\end{array}$ \\
\hline 06 & $\begin{array}{l}\text { Hipertensäo arterial } \\
\text { continuada }\end{array}$ & 06 & $\begin{array}{l}\text { Angústia } \\
\text { ansiedad }\end{array}$ & Liária & 06 & $\begin{array}{l}\text { Pensamento/Fala constante sobre um } \\
\text { mesmo assunto }\end{array}$ \\
\hline 07 & $\begin{array}{l}\text { Problemas dermatológicos } \\
\text { prolongados (pele) }\end{array}$ & 07 & $\begin{array}{l}\text { Hipersen } \\
\text { emotiva }\end{array}$ & iliidade & 07 & Irritabilidade sem causa aparente \\
\hline 08 & Mudança extrema de apetite & 08 & $\begin{array}{l}\text { Perde do } \\
\text { humor }\end{array}$ & so de & & \\
\hline
\end{tabular}

Fonte: Lipp \& Tanganelli; 2002. 


\section{RESULTADOS}

Os dados sociodemográficos da nacionalidade, residência familiar, amostra deste estudo, do qual participaram reprovação, bolsista de pesquisa/ extensão e 120 estudantes, foram apresentados de acordo atividade profissional. com as variáveis: sexo, estado civil,

Tabela 01 - Caracterização das fases do estresse apresentadas pelos acadêmicos de acordo com o Inventário do estresse de Lipp - ISS (n=120). Redenção, Ceará, Brasil, 2019.

\begin{tabular}{lll}
\hline Fases de estresse & $\mathbf{N}$ & $\%$ \\
\hline Alerta & 5 & $4,2 \%$ \\
Resistência & 80 & $66,7 \%$ \\
Exaustão & 9 & $7,5 \%$ \\
Sem estresse & 26 & $21,6 \%$ \\
TOTAL & 120 & $100 \%$ \\
\hline
\end{tabular}

Fonte: dados da própria pesquisa.

A tabela 01 mostra que os discentes entrevistados apresentaram sinais e sintomas do estresse, mas 26 deles $(21,6 \%)$ não apresentaram a quantidade de sintomas necessários para serem classificados em níveis de estresse. No tocante à fase do estresse, houve um predomínio da fase de resistência $80(66,7 \%)$. É preocupante o fato de que 9 alunos $(7,5 \%)$ já se encontram em fase de exaustão.

Tabela 02 - Presença de estresse de acordo com o sexo dos participantes. $(n=120)$. Redenção, Ceará, Brasil, 2019.

Estresse

\begin{tabular}{lccc}
\hline Sexo & Presente & Ausente & Total f (\%) \\
\hline Homens & 47 & 19 & $66(55 \%)$ \\
Mulheres & 39 & 15 & $54(45 \%)$ \\
Total & $86(72 \%)$ & $34(28 \%)$ & $120(100 \%)$ \\
\hline
\end{tabular}

Fonte: dados da própria pesquisa.

A tabela 02 traz os resultados do estudo de $(\mathrm{n}=120 ; 100 \%)$ discentes. Do total, $66(55 \%)$ eram do sexo masculino, deste total $47(71,2 \%)$ apresentaram sintomas de estresse e 19 (28,8\%) não apresentaram, já do sexo feminino participaram 54 (45\%) sendo que 39 $(72,2 \%)$ apresentaram sintomas de estresse e $15(27,8 \%)$ mulheres não apresentaram.

Rev Enferm Atual In Derme v. 95, n. 33, 2021 e-021001 
Tabela 03 - Presença de estresse de acordo com o estado civil dos participantes $(n=120)$. Redenção, Ceará, Brasil, 2019.

Estresse

\begin{tabular}{llll}
\hline Estado civil & Presente & Ausente & $\begin{array}{l}\text { Total } \\
\mathbf{f}(\%)\end{array}$ \\
\hline Solteiro (a) & 64 & 25 & $89(74,2 \%)$ \\
Casado (a) & 21 & 9 & $30(24,2 \%)$ \\
Viúvo (a) & 0 & 0 & $0 \%$ \\
Divorciado (a) & 1 & 0 & $1(1,6 \%)$ \\
\hline Total & $86(72 \%)$ & $34(28 \%)$ & $120(100 \%)$ \\
\hline
\end{tabular}

Fonte: dados da própria pesquisa.

Os dados da tabela 03 mostram que $89(74,2 \%)$ solteiros participaram da pesquisa. Desses 89 discentes, 64 (71,9\%) apresentaram manifestações de estresse, enquanto $25(28,1 \%)$ não apresentaram. Em relação ao público casado, que foram de 30
(24,2\%) pessoas, 21 (70\%) (ou é 17,5\%) delas apresentaram algum tipo de manifestação. Discentes divorciados foram encontrados 1 (1,6\%), mas não foram encontrados participantes em estado de viuvez.

Tabela 04 - Presença de estresse de acordo com a nacionalidade dos estudantes (n=120). Redenção, Ceará, Brasil, 2019.

\begin{tabular}{llll}
\hline Nacionalidade & Presente & Ausente & $\begin{array}{l}\text { Total } \\
\mathbf{f}(\mathbf{\%})\end{array}$ \\
\hline Brasileiros & 39 & 25 & $64(53,3 \%)$ \\
Estrangeiros & 47 & 9 & $56(46,7 \%)$ \\
Total & $86(72 \%)$ & $34(28 \%)$ & $120(100 \%)$ \\
\hline
\end{tabular}

Fonte: dados da própria pesquisa.

Quanto à nacionalidade, a tabela 04 descreve os resultados como: brasileiros 64 $(53,3 \%)$ participaram da pesquisa, $39(60,9 \%)$ apresentaram sintomas de estresse e 25 $(39,1 \%)$ não apresentaram nenhum sintoma.
Quanto aos estrangeiros participaram do estudo $56(46,7 \%)$ alunos, $47 \quad(83,9 \%)$ manifestaram sintomas de estresse e $9(16 \%)$ não manifestaram sintomas. 
Tabela 05 - Presença de estresse de acordo com a residência atual dos participantes $(n=120)$. Redenção, Ceará, Brasil, 2019.

Estresse

\begin{tabular}{llll}
\hline Residência atual & Presente & Ausente & $\begin{array}{l}\text { Total } \\
\mathbf{f}(\boldsymbol{\%})\end{array}$ \\
Redenção/Acarape & 31 & 4 & $35(29,1 \%)$ \\
Outra cidade & 55 & 30 & $85(70,9 \%)$ \\
Total & $86(72 \%)$ & $34(28 \%)$ & $120(100 \%)$ \\
\hline
\end{tabular}

Fonte: dados da própria pesquisa.

Campi da IFES em questão. Desses 85

Do total dos 120 alunos alunos, $55(64,7 \%)$ apresentaram sintomas de participantes do estudo, 85 (70,9\%) deles não moram mais na residência de seus pais, onde tiveram que se deslocar para morar em alguma das cidades onde estão instalados os estresse. E os alunos que ainda moram em suas cidades-natal $35(29,1 \%)$ discentes, 31 $(81,5 \%)$ desses apresentaram sintomas de estresse.

Tabela 06 - Presença de estresse de acordo com reprovações nos currículos dos discentes (n=120). Redenção, Ceará, Brasil, 2019.

Estresse

\begin{tabular}{llll}
\hline Reprovação & Presente & Ausente & $\begin{array}{l}\text { Total } \\
\text { f (\%) }\end{array}$ \\
\hline Sem reprovação & 66 & 14 & $80(67 \%)$ \\
Com reprovação & 20 & 20 & $40(33 \%)$ \\
Total & $86(72 \%)$ & $34(28 \%)$ & $120(100 \%)$ \\
\hline
\end{tabular}

Fonte: dados da própria pesquisa.

Apresentaram manifestações de estresse, em maior número, alunos que não tiveram nenhuma reprovação, ou seja, 62
$(83,7 \%)$. Logo em seguida vêm os alunos que apresentavam de 1 a 3 reprovações, cerca de $17(47,3 \%)$.

Tabela 07 - Presença de estresse de acordo com atividades de pesquisa, extensão e/ou programas similares $(\mathbf{n = 1 2 0})$. Redenção, Ceará, Brasil, 2019.

Estresse

\begin{tabular}{llcl}
\hline Atividades & Presente & Ausente & $\begin{array}{l}\text { Total } \\
\mathbf{f}(\boldsymbol{\%})\end{array}$ \\
\hline SIM & 32 & 15 & $47(39,16 \%)$ \\
NÃO & 54 & 19 & $73(60,84 \%)$
\end{tabular}


Total

$86(72 \%)$

$34(28 \%)$

$120(100 \%)$

Fonte: dados da própria pesquisa.

Do total estudado, $47 \quad(39,16 \%)$ possuíam algum tipo de bolsa de pesquisa, extensão ou outros programas. Desses 47 alunos, 32 (26,66\%) apresentaram algum nível de manifestação de estresse, número bastante elevado nesse tipo de público. No entanto, dos que não realizam atividades de pesquisa, $73(60,84 \%)$ apresentam estresse.

Tabela 08- Presença de estresse de acordo com atividade profissional/vínculo empregatício (n= 120). Redenção, Ceará, Brasil, 2019.

\begin{tabular}{llll}
\hline $\begin{array}{l}\text { Atividade } \\
\text { profissional }\end{array}$ & Presente & Ausente & $\begin{array}{l}\text { Total } \\
\mathbf{f}(\%)\end{array}$ \\
\hline SIM & 4 & 3 & $7(5,83 \%)$ \\
NÃO & 82 & 31 & $113(94,16 \%)$ \\
Total & $86(72 \%)$ & $34(28 \%)$ & $120(100 \%)$ \\
\hline
\end{tabular}

Fonte: dados da própria pesquisa.

Dos 120 alunos estudados, apenas 7 $(5,83 \%)$ desempenhavam alguma atividade empregatícia, contra $113(94,16 \%)$ que não exerciam. Desses 7 universitários, $4(57,1 \%)$ apresentaram manifestações de estresse, mas foi um índice muito próximo aos alunos que não apresentaram estresse.

\section{DISCUSSÃO}

Atualmente, vive-se um tempo de grandes exigências de realizações pessoais e profissionais, além de demandas sociais das mais variadas, gerando muitas e difíceis tomadas de decisões. Além disso, o avanço tecnológico exige do organismo humano constantes processos de readaptações psicofisiológicas que contribuem para 0 aumento do número de pessoas com sintomas de estresse. Pesquisas têm mostrado que o Brasil é o segundo país que mais apresenta sintomas de estresse no mundo ${ }^{(9)}$.

O estresse pode ser definido como uma reação muito complexa, composta de alterações psicofisiológicas que ocorrem quando o indivíduo é forçado a enfrentar situações que ultrapassam sua habilidade de enfrentamento. As situações que provocam ou conduzem ao estresse são denominadas estressores e são agrupadas como: eventos de vida estressores, acontecimentos diários menores e situações de tensão crônica ${ }^{(11)}$.

A vida de um estudante universitário, em geral, é marcada por um processo de mudanças e adaptações. Esta fase também é caracterizada por uma série de exigências e necessidades provenientes da 
grade curricular da instituição de ensino. No prazos para entrega de trabalhos, provas, relatórios de estágios, apresentação de seminários e preparação do trabalho de conclusão de curso que deve ser entregue e apresentado oralmente. Diante dessas obrigações, o acadêmico, especialmente no último período, está sujeito a diversas reações emocionais, caracterizando, assim, um grande período de estresse ${ }^{(7)}$.

Nesta pesquisa verificou-se que dos 120 alunos participantes do estudo, 94 discentes apresentaram manifestações de estresse em uma das categorias, (alerta, resistência e exaustão), especialmente a categoria resistência (fase 2$)(80 ; 66,7 \%)$ foi predominante; e $(26 ; 21,6 \%)$ não apresentaram manifestações de estresse. Esta ocorrência pode estar relacionada a atividades da grade curricular, carga horária de trabalho e ainda distância dos familiares e do $\operatorname{lar}^{(12)}$. É provável que os alunos que se encontram nesta fase de manifestação de estresse tenham algum comprometimento no seu processo de aprendizagem e na sua qualidade de vida, dado que na fase de resistência existem significativos desgastes físicos e mentais e tentativas do organismo de reequilíbrio das funções fisiológicas frente ao processo de somatização.

O próprio organismo busca maneiras de se livrar desses sinais desgastantes, tenta resistir aos malefícios provocados por essas manifestações desses âmbito acadêmico o aluno tem sintomas, buscando retornar ao estado de equilíbrio ou homeostase. Sendo esta a fase intermediária do estresse, indica que os discentes estão buscando a adaptação e recursos para enfrentar situações estressoras $^{(13)}$.

A pesquisa mostrou, ainda, que os sintomas de estresse foram predominantes tanto em homens quanto em mulheres. Justificado pelo fato que os discentes estão lidando com novas rotinas $\mathrm{e}$ as manifestações dos sintomas de estresse são bem frequentes nas variáveis estudadas devido às obrigações enquanto $\operatorname{acadêmicos}^{(2)}$.

Vale ressaltar que as incertezas quanto ao mercado de trabalho e a busca por ser um profissional mais qualificado para alcançar um bom emprego ao fim da graduação, pode gerar nos acadêmicos um sentimento de necessidade de aproveitar todas as oportunidades disponíveis, gerando uma sobrecarga de atividades e o excesso de atividades de estudo, em detrimento de sua vida social e familiar.

Do total dos 120 alunos participantes do estudo, 85 deles não moram mais na residência de seus pais, pois, tiveram que se deslocar para morar em alguma das cidades onde estão instalados nos Campi da IFES em questão. Um estudo realizado na universidade de Campinas com 150 estudantes, evidenciou que $89,3 \%$ dos 
estudantes que não estavam em seu lugar de origem se consideram estressados ${ }^{(14)}$.

Levando em consideração a nacionalidade, o público discente que mais apresentou a ocorrência de manifestações foram os alunos estrangeiros. Diante deste resultado, podemos dizer que as causas para a manifestação de estresse neste público podem ser: mudança abrupta de lugar de origem, distância da família e de seus entes, choque cultural, adequação a um novo ambiente que é a universidade, dentre vários outros motivos $^{(15)}$.

No presente estudo, o estresse também foi muito frequente entre os estudantes brasileiros que mudaram de cidade para ingressar em um curso de graduação. A saída da casa dos pais pode ser considerada um fator desencadeante do estresse, muitos discentes para ingressarem na universidade, tem que migrar para o lugar onde se encontra a instituição de ensino. Com esta mudança, distância dos familiares, convivência com novas pessoas e a responsabilidade de viver em uma casa com colegas, bem como os custos financeiros de ficar em uma outra moradia, geram fatores estressores e consequentemente, os sintomas do estresse.

Além disso, diante do calendário acadêmico e da grade curricular de ensino, o discente se mostra preocupado em seguir todas as responsabilidades que esta lhe impõe, daí surgem as cobranças de si próprio, e a dedicação aos estudos. Estes podem ser considerados fatores que podem desencadear o estresse e seus sintomas ${ }^{(12)}$. Podemos considerar particularmente as especificidades das atividades que esses alunos estariam desempenhando, tais como: confecção de Trabalho de Conclusão do Curso (TCC), ansiedade em relação a formatura, estágios curriculares, dentre $\operatorname{outros}^{(9)}$.

O estudo mostra que apresentaram manifestações de estresse em maior número, alunos que não tiveram nenhuma reprovação (80 alunos). Podemos relacionar este número ao fato de o aluno conseguir conciliar suas atividades curriculares e sua vida pessoal, porém, diante das responsabilidades o acadêmico pode desencadear o estresse devido ao compromisso de manter o seu histórico acadêmico livre de reprovações. Podemos relacionar este fator ao estresse positivo, que é quando o indivíduo consegue centrar nos seus objetivos mesmo sob $\operatorname{pressão}^{(2)}$.

De um total de 47 alunos que possuem bolsa de pesquisa e extensão, 32 apresentaram sintomas de estresse. Podemos fazer ligação ao fato de, além de ter que estudar e não apresentar reprovações, esses públicos ainda têm que desempenhar atividades extracurriculares pertinentes a bolsa a qual é vinculado, dessa maneira, esse aluno tem sua carga de estudos maior que um aluno regular que não seja bolsista. 
$\mathrm{O}$ vínculo empregatício e os estudos, juntos podem desencadear sintomas de estresse. A pesquisa mostra que dos 120 alunos estudados, apenas 7 desempenhavam alguma atividade empregatícia, contra 113 que não exerciam. Desses 7 estudantes, 4 apresentaram manifestações de estresse. $\mathrm{O}$ fato de poucos alunos possuírem emprego, está relacionada ao estudo em regime integral, pois, como o discente passa o dia todo na universidade não pode exercer alguma atividade extra, exceto aquele aluno que estuda no período noturno e que pode usar suas horas durante o dia para trabalha ${ }^{(2)}$. O estresse sendo caracterizado por uma desordem emocional gerando sintomas físicos e ou psicológicos, quando o indivíduo entra em contato com algum fator estressor pode trazer sintomas crônicos que são tratados a partir do uso de medicamentos conforme prescrição médica, como exemplo disso temos a ansiedade que pode ser minimizada a partir do uso de ansiolíticos ${ }^{(12)}$.

É importante destacar que o estresse pode ser desencadeado pelo excesso de atividades, que é o que parece estar acontecendo com os estudantes participantes deste estudo, ao mesmo tempo em que ele é prejudicial ao aprendizado. Isso gera um ciclo vicioso em que a necessidade de realização de atividades acadêmicas gera estresse e o estresse dificulta que as atividades sejam realizadas, aumentando a carga de estresse e podendo comprometer a qualidade de vida dos mesmos.

Este estudo teve como limitações a dificuldade de encontrar os alunos nos seus intervalos de atividades acadêmicas, considerando que os mesmo utilizam tais horários para suas refeições e descanso. Dessa forma, é importante a realização de novos estudos que investiguem a qualidade de vida dos estudantes, bem como todas as suas nuances, visando identificar objetivamente os pontos passíveis de intervenção para que se possa reduzir os níveis de estresse entre estudantes de ensino superior.

Considerando o estudo realizado e resultados obtidos, percebe-se a necessidade da realização de ações de promoção à saúde, por parte da IFES estudada, com o objetivo de prevenir agravos emocionais e possíveis distúrbios psiquiátricos como ansiedade e depressão nos estudantes; contribuindo, dessa forma, para o bem-estar biopsicossocial destes e minimizar o impacto do estresse, que para alguns autores é considerado uma síndrome evolutiva com três fases distintas ${ }^{(16)}$.

\section{CONCLUSÕES}

Com base nos resultados apresentados, o estudo demonstrou que o perfil das manifestações de estresse é consideravelmente alto nos discentes da instituição estudada e pode estar classificado, especialmente, na fase intermediária (resistência); este achado pode indicar um grande impacto na qualidade de vida dos Rev Enferm Atual In Derme v. 95, n. 33, 2021 e-021001 
discentes, podendo até comprometer o processo de aprendizagem dos mesmos.

\section{REFERÊNCIAS}

1. Assis CL, Silva APF, Lopes MS, Silva PCB, Santini TO. Sintomas de estresse em concluintes do curso de psicologia de uma faculdade privada do norte do país. Advances in Health Psycology [Internet] 2013 [acesso em 15 ago 2018] 21(1):23-28. Disponível em: https://www.metodista.br/revistas/revistasims/index.php/MUD/article/view/3668/3644.

2. Santos FS, Maia CRC, Faedo FC, Gomes GPC, Nunes ME, Oliveira MVM. Estresse em estudantes de cursos preparatórios e de graduação em medicina. Revista Brasileira de Educação Médica [Internet] 2017 [acesso em 15 ago 2018] 41(2)194-200. Disponível em: https://www.scielo.br/pdf/rbem/v41n2/19815271-rbem-41-2-0194.pdf.

3. Rovida TAS, Sumida DH, Santos AS, Moimaz SAS, Garbin CAS. Estresse e o estilo de vida dos acadêmicos ingressantes em um curso de graduação em Odontologia. Revista da ABENO [Internet] 2015 [acesso em 15 ago 2018] 15(3)26-34. Disponível em: http://revodonto.bvsalud.org/pdf/abeno/v15n3 la04v15n3.pdf.

4. Cruz MZ, Júnior AP. Corpo, mente e emoções: referenciais teóricos da psicossomática. Rev. Simbio- logias [Internet] 2011 [acesso em 22 ago 2018] 4(6)46-69. Disponível em: https://www.ibb.unesp.br/Home/ensino/depart amentos/educacao/revistasimbiologias/corpo-mente-e-emocoes.pdf.

5. Lima LR, Soares MEC, Prado SN, Albuquerque GSC. Estresse do estudante de medicina e rendimento acadêmico. Revista Brasileira de Educação Médica [Internet]. 2016 [acesso em 03 set 2018] 40 (4) 678-684. Disponível em: https://www.scielo.br/pdf/rbem/v40n4/19815271-rbem-40-4-0678.pdf.
6. Pagliarone AC, Sforcin JN. Estresse: revisão sobre seus efeitos no sistema imunológico. Biosaúde [Internet] 2009 [acesso em 03 set 2018] 11(1)57-90. Disponível

em: http://www.uel.br/revistas/uel/index.php/bios aude/article/view/24304/17878.

7. Silva JA, Marra AV. Estresse em estudantes de administração: um estudo na Universidade Federal de Viçosa. Revista de administração da UEG [Internet] 2017 [acesso em 14 set 2018] 8(3) 74-89. Disponível em: https://www.revista.ueg.br/index.php/revista administracao/article/view/5750.

8. Pirajá GA, Sousa TF, Fonseca AS, Barbosa AR, Nahas MV. Auto avaliação positiva de estresse e prática de atividades físicas no lazer em estudantes universitários brasileiros. Rev Bras Ativ Fis Saúde [Internet] 2013 [acesso em 28 out 2018] 18(6)740-749. Disponível em: file:///C:/Users/ALBERTO\%20M'BATNA/D esktop/Piraja_etal_2013_APestresseeAFL_M ONISA2010.pdf.

9. Lilla M, Tuleta ID, Amaral JB, Souza PC, França $\mathrm{CN}$. Estresse em estudantes do quarto ano de graduação expostos à realização do trabalho de conclusão de curso. Revista Brasileira de Ciências da Saúde [Internet]. 2017 [acesso em 10 nov 2018] 21(4)307- 312. Disponível em: https://periodicos.ufpb.br/ojs/index.php/rbcs/a rticle/view/307-312/18649.

10. Lipp MEN, Tanganelli MS. Stress e qualidade de vida em magistrados da justiça do trabalho: diferenças entre homens e mulheres. Psicologia: reflexão e crítica. 2002; 15(3): 537-48.

11. Peleias IR, Guimarães ER, Chan BL, Carlotto MS. A síndrome de Burnout em estudantes de ciências contábeis de IES Privadas: pesquisa na cidade de São Paulo. Revista de Educação e Pesquisa em Contabilidade [Internet] 2017 [acesso em 10 nov 2018] 11(1)30-51. Disponível em: 
http://www.repec.org.br/repec/article/view/1 468/1207.

12. Cestari VRF, Barbosa IV, Florêncio RS, Pessoa VLMP, Moreira TMM. Estresse em estudantes de enfermagem: estudo sobre vulnerabilidades sociodemográficas e acadêmicas. Rev. Acta Paul Enferm [Internet] 2017 [acesso em 10 nov 2018] 30(2)190-6. Disponível em: https://www.scielo.br/pdf/ape/v30n2/19820194-ape-30-02-0190.pdf .

13. Preto VA, Benevides MS, Queiroz BG, Pereira SS, Souza BOP, Sailler GC et al. Estresse e características sociodemográficas em universitários de enfermagem. Rev enferm UFPE on line [Internet] 2018 [acesso em 20 nov 2018] 12(3)701-7. Disponível em: https://periodicos.ufpe.br/revistas/revistaenfer magem/article/view/231060/28028.

14. Lameu JN, Salazar TL, Souza WF, . Prevalência de sintomas de stress entre graduandos de uma universidade pública. Psicologia da Educação [Internet] 2016 [acesso em 26 set 2018] 42 13-22. Disponível em: https://revistas.pucsp.br/psicoeduca/article/vie $\underline{\mathrm{w} / 30548 / 21130}$.
15. Girardi JF, Borges LM. Dimensões do sofrimento psíquico em estudantes universitários estrangeiros. Psico [Internet] 2017 [acesso em $30 \mathrm{dez} 2018$ ] 48(4)256-263. Disponível em: https://revistaseletronicas.pucrs.br/ojs/index.p hp/revistapsico/article/view/26143/pdf.

16. Bezerra CMB, Silva KKM, Aquino ASF, Martino MMF. Instrumentos verificadores de estresse e da síndrome de burnout: revisão integrativa. Revista Enfermagem Atual [Internet] 2016 [acesso em $24 \mathrm{dez} 2020] 79$. Disponível em: https://revistaenfermagematual.com.br/index. php/revista/article/view/339/223.

Submissão: 2020-09-22

Aprovado: 2021-01-04 